\title{
SUPERNOVA REMNANTS IN MOLECULAR CLOUDS
}

\author{
Roger A. Chevalier \\ Department of Astronomy, University of Virginia, P.O. Box 3818 \\ Charlottesville, VA 22903; rac5x@virginia.edu
}

\begin{abstract}
Massive $\left(\gtrsim 8 M_{\odot}\right)$ stars may end their lives in the molecular clouds in which they were born. O-type stars probably have sufficient photoionizing radiation and wind power to clear a region $>15 \mathrm{pc}$ in radius of molecular material. Early B stars (B1-B3 on the main sequence, or $8-12 M_{\odot}$ stars) are not capable of this and may interact directly with molecular gas. Molecular clouds are known to be clumpy, with dense molecular clumps occupying only a few percent of the volume. A supernova remnant then evolves primarily in the interclump medium, which has a density $n_{H}=5-25 \mathrm{H}$ atoms $\mathrm{cm}^{-3}$. The remnant becomes radiative at a radius of $\sim 6 \mathrm{pc}$, forming a shell that is magnetically supported. The structure of the shell can be described by a self-similar solution. When this shell interacts with the dense clumps, the molecular shock fronts are driven by a considerable overpressure compared to the pressure in the rest of the remnant. The expected range of clump sizes leads to a complex velocity distribution, with the possibility of molecular gas accelerated to a high velocity. Observations of the remnants W44 and IC 443 can be understood in this model. W44 has a shell expanding at $\sim 150 \mathrm{~km} \mathrm{~s}^{-1}$ expanding into a medium with density $4-5 \mathrm{~cm}^{-3}$. The shock emission expected in such a model is consistent with the observed $\mathrm{H} \alpha$ surface brightness and the [OI] $63 \mu \mathrm{m}$ line luminosity. The clump interaction is seen in $\mathrm{OH}$ maser emission, which shows a magnetic field strength that is consistent with that expected in the model. IC 443 appears to be expanding at a lower velocity, $100 \mathrm{~km} \mathrm{~s}^{-1}$, into an interclump medium with a higher density, $\sim 15 \mathrm{~cm}^{-3}$. The interaction of the radiative shell with molecular clumps can produce the molecular emission that is observed from IC 443. Both remnants are shell sources of radio synchrotron emission, which can be attributed to relativistic electrons in the cool radiative shell. If ambient cosmic ray electrons are further accelerated by the shock front and by the postshock compression, the radio fluxes and the flat spectral indices of W44 and IC 443 can be explained. The energetic electrons are in a high density shell and their bresstrahlung emission can approximately produce the $\gamma$-ray fluxes observed by EGRET. Molecular clouds have a significant uniform magnetic field component so that heat conduction is likely to be important in the hot interior and can explain the isothermal X-ray emission observed from the remnants. Evaporation of the dense clumps by conduction is unlikely to be a dominant process.
\end{abstract}


Subject headings: Acceleration of Particles — ISM: Clouds — ISM: Molecules — ISM: Supernova Remnants

\section{INTRODUCTION}

Twenty years ago DeNoyer (1979a,b) found clear evidence for the interaction of the supernova remnant IC 443 with molecular gas, based on observations of disturbed CO emission. IC 443 has become an especially rich target for molecular line observations, including $\mathrm{H}_{2}$ (Burton et al. 1988; Richter et al. 1995b); $\mathrm{CO}$ and $\mathrm{HCO}^{+}$(Dickman et al. 1992; Tauber et al. 1994); HCN, CN, $\mathrm{SiO}, \mathrm{CS}, \mathrm{SO}, \mathrm{H}_{2} \mathrm{CO}$, and $\mathrm{C}_{2} \mathrm{H}$ (Turner et al. 1992; van Dishoeck et al. 1993). Weaker evidence for interaction was found in CO observations of W44 and W28 (Wootten 1977, 1981), which were earlier found to be sources of $\mathrm{OH} 1720 \mathrm{MHz}$ line emission (Goss \& Robinson 1968). OH 1720 $\mathrm{MHz}$ maser line emission has emerged as a signpost of molecular interaction with the detection of compact $\mathrm{OH}$ emission from 8 supernova remnants (Frail et al. 1996), including W44, IC 443, and 3C391. These remnants typically have a radius $\sim 10 \mathrm{pc}$.

The interaction of supernova remnants with molecular clouds can plausibly occur because massive stars are born in such clouds and have a relatively short lifetime. Stars with initial mass ¿8 $M_{\odot}$ are expected to end their lives as core collapse supernovae. Previous theoretical treatments of the interaction problem have concentrated on the interaction with very dense cloud material. Shull (1980) considered an ambient medium with density $n_{o}=10^{5} \mathrm{~cm}^{-3}$ and predicted strong IR (infrared) emission that decayed after 20 years. Wheeler, Mazurek, \& Sivaramakrishnan (1980) found that evolution in a medium with $n_{o}=10^{4} \mathrm{~cm}^{-3}$ leads to a high IR luminosity for $\sim 100$ years, followed by a rapid drop. The remnant radius is $\sim 1 \mathrm{pc}$ during its bright phase. Events of this kind have not been identified, but it is not clear what limits can be set from existing IR observations. The existing observations of remnant/cloud interactions require a different model.

The model I explore here is the interaction of a remnant with a clumpy molecular cloud. Observations of molecular clouds have shown them to be clumpy, with a filling factor for the dense molecular clumps of $\sim 0.02-0.08$ (Blitz 1993). The interclump gas has a density of $5-25 \mathrm{~cm}^{-3}$. A supernova is more likely to explode in the interclump gas than in a dense clump, especially when the effects of presupernova winds and photoionization and stellar motion are taken into account. In $\S 2$, I consider the likely environment for a supernova in a molecular cloud, including the effects of presupernova winds and photoionization as well as the structure of the molecular cloud. The remnant expansion in a molecular cloud is treated in $\S 3$, on the assumption that the evolution in the interclump region can be separated from the interaction with clumps. In $\S 4$, I compare this

model to observations of three well-observed remnants: W44, IC 443, and 3C391. The model does appear to be compatible with the basic observations. Nonthermal particles and their emission are treated in $\S 5$. This topic is of special interest in view of the probable detection of supernova 
remnants in molecular clouds with the EGRET experiment on the CGRO (Compton Gamma-Ray Observatory) (Esposito et al. 1996). Possible future work is briefly discussed in $\S 6$.

\section{THE SUPERNOVA ENVIRONMENT}

Observational studies of molecular clouds have shown them to have dense molecular clumps embedded in a lower density interclump medium (Blitz 1993). A good example is the study of the Rosette cloud by Williams, Blitz, \& Stark (1995), who show that the clumps have a density of $440 \mathrm{H}$ atoms $\mathrm{cm}^{-3}$ and a filling factor of 0.08 . The interclump medium has a density of 11 $\mathrm{H}$ atoms $\mathrm{cm}^{-3}$, so that the clumps comprise 3.5 times more mass than the interclump medium, but occupy a small fraction of the volume. The distribution of clump masses, $M$, in clouds can be described by a power law, $d N(M) \propto M^{-1.54} d M$, where $d N$ is the number of clouds with mass between $M$ and $M+d M$. The relevant mass range is $1-3,000 M_{\odot}$, which corresponds to diameters of $0.2-2.2 \mathrm{pc}$ if clumps have a typical $\mathrm{H}_{2}$ density of $10^{3} \mathrm{~cm}^{-3}$. More generally, Blitz (1993) notes some properties of typical GMC's (Giant Molecular Clouds): total mass $\sim 10^{5} M_{\odot}$, diameter $\sim 45 \mathrm{pc}, \mathrm{H}_{2}$ density in clumps of $10^{3} \mathrm{~cm}^{-3}$, interclump gas density in the range $5-25 \mathrm{H}$ atoms $\mathrm{cm}^{-3}$, and clump filling factor of $2-8 \%$. The clump mass spectrum can be described by $d N(M) \propto M^{-q} d M$ with $q$ in the range $1.4-1.9$. More recently, Kramer et al. (1998) have also found power law distributions of clump mass with $q \approx 1.6-1.8$ in 7 clouds; the clump masses range from $10^{-4}-10^{3} M_{\odot}$. With this mass spectrum, most of the clouds are small, but most of the mass is in large clumps. The lower limit to the clump masses is set by observational constaints. Blitz (1993) noted that the clump shapes vary from spherical to highly irregular.

An interclump pressure, $p / k \approx 10^{5} \mathrm{~K} \mathrm{~cm}^{-3}$, is needed to confine the dense clumps and to support the cloud against gravitational collapse (Blitz 1993). The thermal pressure in the interclump gas is clearly too small and magnetic fields are a plausible pressure source. Studies of the polarization of the light from stars behind molecular clouds implies that the interclump magnetic field does have a relatively smooth structure (Heiles et al. 1993). Setting $B_{o}^{2} / 8 \pi$ equal to the above pressure yields $B_{o}=19 p_{5}^{1 / 2} \mu \mathrm{G}$, where $B_{o}$ is the uniform field component and $p_{5}$ is $p / k$ in units of $10^{5} \mathrm{~K} \mathrm{~cm}^{-3}$. However, a uniform magnetic field would not provide support along the magnetic field and would not explain the large line widths observed in clouds. An analysis of the polarization and Zeeman effect in the dark cloud L204 shows consistency with equal contributions to the pressure from a uniform field and a fluctuating, nonuniform component (Myers \& Goodman 1991; Heiles et al. 1993). The uniform component is then $B_{o}=13 p_{5}^{1 / 2} \mu \mathrm{G}$. The magnetic field strength deduced in L204 is consistent with this value.

The molecular cloud can be influenced by stellar mass loss and photoionization by the progenitor star before the supernova. The effect of these processes can be to remove molecular gas from the vicinity of the central star. From the point of view of interpreting the observations of supernova remnants mentioned in $\S 1$, it is important whether molecular clumps can survive within $\sim 15$ pc of the central star. McKee, Van Buren, \& Lazareff (1984) found that B0-O4 stars 
could clear a region $28 \mathrm{pc}$ in radius of clumps if the mean density was $n_{m}=10 \mathrm{~cm}^{-3}$. Small clumps are completely photoevaporated and large clumps move out by the "rocket" effect. If the scaling for the radius $\propto n_{m}^{-0.3}$ (McKee et al. 1984) can be extrapolated to the somewhat higher mean densities of interest here, a region $\gtrsim 15 \mathrm{pc}$ is expected to become free of molecular clumps. The photoevaporation of clumps has been studied in greater detail by Bertoldi \& McKee (1990), who found that the rocket effect is smaller than that in previous investigations. However, the rocket velocity $\gtrsim 5 \mathrm{~km} \mathrm{~s}^{-1}$ is still sufficient to clear a region $\gtrsim 15 \mathrm{pc}$ in size. The additional effect of stellar winds is to clear a wind bubble in the homogenized region for the stronger winds or to create a wind bubble within the HII region (McKee et al. 1984).

The situation changes for early B type stars, which have weaker photoionizing fluxes and stellar winds. These stars have sufficiently long lifetimes that their HII regions can come into pressure equilibrium with the surrounding medium. I assume that the surrounding interclump medium exerts a pressure $p / k=10^{5} \mathrm{~K} \mathrm{~cm}^{-3}$ and that the gas temperature in the HII region is $10^{4} \mathrm{~K}$, so that the density in the ionized gas is $\sim 5 \mathrm{H}$ atoms $\mathrm{cm}^{-3}$. For the ionizing fluxes given in Panagia (1973), Table 1 shows the radii of the ionized regions, $R_{\text {ionized }}$, for O9-B3 main sequence stars. The small sizes of the ionized regions for B1-B3 stars is evident. The radii for the regions around the O9 and B0 stars appears to be smaller than those obtained by McKee et al. (1984); this is presumably because of the higher surrounding pressure taken here.

Stellar winds are an additional effect on the circumstellar environment. The maximum size, $R_{b}$, of the wind bubble is that at which the bubble comes into pressure equilibrium with its surroundings, or the wind energy production is about equal to the displaced energy:

$$
\frac{1}{2} \dot{M} v_{w}^{2} \tau_{m s}=\left(\frac{4}{3} \pi R_{b}^{3}\right) \frac{3}{2} p_{o}
$$

where $\dot{M}$ and $v_{w}$ are the wind mass loss rate and velocity and $\tau_{m s}$ is the main sequence age. For typical parameters, the result for $R_{b}$ is

$$
R_{b}=5.8\left(\frac{\dot{M}}{10^{-8} M_{\odot} \mathrm{yr}^{-1}}\right)^{1 / 3}\left(\frac{v_{w}}{700 \mathrm{~km} \mathrm{~s}^{-1}}\right)^{2 / 3}\left(\frac{p / k}{10^{5} \mathrm{~K} \mathrm{~cm}^{-3}}\right)^{-1 / 3}\left(\frac{\tau_{m s}}{10^{7} \mathrm{yr}}\right)^{1 / 3} \mathrm{pc} .
$$

The values of $\dot{M}$ in Table 1 are from de Jager, Nieuwenhuijzen, \& van der Hucht (1988). The values of $\tau_{m s}$ are estimated from the evolutionary tracks of Schaller et al. (1992) for a somewhat different set of stellar masses. I have taken $v_{w}=700 \mathrm{~km} \mathrm{~s}^{-1}$, as appropriate for these stellar types, and $p_{o} / k=10^{5} \mathrm{~K} \mathrm{~cm}^{-3}$ in order to obtain the values of $R_{b}$ shown in Table 1 . It can be seen that the wind bubble is somewhat larger than the ionized region, although B1-B3 stars do have bubble sizes that are smaller than those of the remnants of interest. McKee et al. (1984) note that if the wind bubble begins to expand beyond the ionized region, matter will be photoevaporated from clumps and may mix with the hot gas inside the bubble. The higher density gas can have a cooling time that is shorter than the age, so that the assumption of energy conservation of the bubble gas made in eq. (1) is not valid, and the bubble radius may be limited to a value close to that of the ionized region. 
In the later stages of evolution, the star becomes a red supergiant and has a slow dense wind which can cool after passing through a termination shock. Equating the wind ram pressure $\left(\rho_{w} v_{w}^{2}\right)$ with the interclump pressure, $p_{o} / k=10^{5} \mathrm{~K} \mathrm{~cm}^{-3}$, yields an approximate stopping radius of

$$
r_{s t}=0.62 \dot{M}_{-5}^{1 / 2} v_{w 1}^{1 / 2} \quad \mathrm{pc},
$$

where $\dot{M}_{-5}$ is the wind mass loss rate in units of $10^{-5} \quad M_{\odot} \mathrm{yr}^{-1}$ and $v_{w 1}$ is the wind velocity in units of $10 \mathrm{~km} \mathrm{~s}^{-1}$.

Even if the immediate progenitor star does not modify its environment, it is quite possible that more massive stars form in association with the progenitor and that these stars affect the environment. The $8-12 M_{\odot}$ stars explode relatively late so that more massive companions do have an opportunity to change the environment. However, the lower mass molecular clouds (mass $\sim 10^{4} M_{\odot}$ ) can have one or no O stars (Williams \& McKee 1997). This discussion leads to the conclusion supernova remnants with radii $\sim 10$ pc can involve interaction with a quiescent molecular cloud, although they are a fraction of the Type II supernovae. The examination of remnants where cloud interaction appears to be taking place will then indicate whether this is occurring, or whether modification of the supernova surroundings by the progenitor star and its companions needs to be taken into account.

\section{SUPERNOVA EXPANSION IN A MOLECULAR CLOUD}

\subsection{Expansion in the Interclump Medium}

A reference interclump density is $n_{o}=10 \mathrm{H}$ atoms $\mathrm{cm}^{-3}$. I assume that as a first approximation, the presence of the dense clumps can be neglected in the propagation of the supernova shock front. The evolution of a supernova remnant in a uniform medium has been frequently treated, so I primarily highlight some of the aspects that are important for the molecular cloud case.

In the first phase of evolution, the supernova ejecta are decelerated by interaction with the ambient medium. The ejecta are expected to be mostly freely expanding until they have swept up their own mass, which occurs at a radius $R_{m}=1.9 M_{1}^{1 / 3} n_{1}^{-1 / 3} \mathrm{pc}$, where $M_{1}$ is the ejected mass in units of $10 M_{\odot}$ and $n_{1}$ is the interclump $\mathrm{H}$ density in units of $10 \mathrm{H}$ atoms $\mathrm{cm}^{-3}$. This radius is sufficiently small that the effects of the progenitor star on its surroundings are likely to be important for this initial phase of evolution. However, once the shock front has propagated several pc, the flow in the interclump medium should tend toward the Sedov blast wave solution.

The remnant next enters a radiative phase (Cox 1972; Chevalier 1974; Cioffi, McKee, \& Bertschinger 1988). For gas with solar metallicity, Cioffi et al. (1988) find that the time taken to cool to 0 temperature, i.e. shell formation, is $t_{s f}=9.7 \times 10^{3} E_{51}^{3 / 14} n_{1}^{-4 / 7}$ years, where $E_{51}$ is the supernova energy in units of $10^{51}$ ergs. The effects of radiative losses actually become 
important at an earlier time and Cioffi et al. suggest that the pressure-driven snowplow (PDS) phase begins at at $t_{\mathrm{PDS}}=t_{s f} / e=3.6 \times 10^{3} E_{51}^{3 / 14} n_{1}^{-4 / 7}$ years, where $e$ is the base of the natural logarithm. The corresponding shock radius and velocity are $R_{\mathrm{PDS}}=5.2 E_{51}^{2 / 7} n_{1}^{-3 / 7} \mathrm{pc}$ and $v_{\mathrm{PDS}}=574 E_{51}^{1 / 14} n_{1}^{1 / 7} \mathrm{~km} \mathrm{~s}^{-1}$. For the further evolution of the remnant radius, Cioffi et al. (1988) advocate an offset power law in time. However, over the range of $(5-50) t_{\text {PDS }}$ the value of $v_{s} t / R$ is in the range $0.31-0.33$ so that a power law in time is an adequate representation.

For $p / k=10^{5} \mathrm{~cm}^{-3} \mathrm{~K}$ and $n_{o}=10 \mathrm{~cm}^{-3}$, the isothermal sound speed in the cloud is $7.7 \mathrm{~km} \mathrm{~s}^{-1}$. For $E_{51}=1$ and $n_{1}=1$, the shock velocity drops to twice the isothermal sound speed at a radius of 25 pc. Because the typical diameter of a cloud is 45 pc (Blitz 1993), the remnant is more likely to break out of the cloud before it merges with the cloud.

\subsection{Radiative Shell Structure}

The expansion of the radiative shell can be treated independently of the shell structure, which depends on the radiative cooling and on contributions to the pressure. This is true provided that the shell thickness is small compared to its radius $(\Delta R / R \ll 1)$. Bertschinger (1986) developed an analytical solution for the shell structure on the assumption of radiative cooling to a particular temperature and adiabatic evolution after that point; magnetic effects were neglected. Cioffi et al. (1988) made the same assumption in their numerical calculations, allowing radiative cooling to $1.2 \times 10^{4} \mathrm{~K}$. On the other hand, Chevalier (1974) and Slavin \& Cox (1992) performed numerical calculations with magnetic support in the dense shell.

The observations of molecular clouds point to the presence of a significant uniform magnetic field. I take the case of expansion in a uniform magnetic field and start with the expansion perpendicular to the magnetic field direction. If a uniform gives a pressure of $p / k=10^{5} \mathrm{~cm}^{-3} \mathrm{~K}$, the field strength is $1.9 \times 10^{-5} \mathrm{G}$. The field is likely to have a significant nonuniform component, but a tangential field component $\sim 10^{-5} \mathrm{G}$ is plausible. The density at the point where the magnetic pressure becomes important can be expressed as $n_{m}=2.4 \times 10^{2} v_{2} n_{1}^{3 / 2} B_{\text {ot-5 }}^{-1} \mathrm{~cm}^{-3}$, where $v_{2}$ is the shock velocity in units of $100 \mathrm{~km} \mathrm{~s}^{-1}$ and $B_{o t-5}$ is the ambient tangential field in units of $10^{-5} \mathrm{G}$ (e.g., Draine \& McKee 1993). The immediate postshock temperature is $1.38 \times 10^{5} v_{2}^{2} \mathrm{~K}$ if the gas is fully ionized, so that the tangential field comes to dominate the pressure provided that the temperature drops below $3 \times 10^{4} v_{2} n_{1}^{-1 / 2} B_{o t-5} \mathrm{~K}$; the radiative cooling is certainly likely to take the temperature below this value and the cooling becomes more isochoric than isobaric as in the case of a lower magnetic field. The magnetic field strength after the compression is $B=2.4 \times 10^{-4} n_{1}^{1 / 2} v_{2}$ G. If we consider a ring of radius $R$ and thickness $\Delta R$ perpendicular to the field direction, flux conservation implies that the quantity $B_{t} 2 \pi R \Delta R$ is conserved. Mass conservation implies $\rho 4 \pi R^{2} \Delta R$ is conserved, for spherical expansion. The result is $B_{t} \propto \rho R$. This is an approximation because the expansion is not exactly spherical.

The method of Bertschinger (1986) for the shell structure can be extended to the magnetic 
case of interest here. The equations of mass and momentum conservation for spherical flow are

$$
\frac{d \rho}{d t}+\frac{\rho}{r^{2}} \frac{\partial r^{2} v}{\partial r}=0, \quad \rho \frac{d v}{d t}+\frac{1}{8 \pi} \frac{d B_{t}^{2}}{d r}+\frac{B_{t}^{2}}{4 \pi r}=0,
$$

where $d / d t=\partial / \partial t+v \partial / \partial r$ and the magnetic pressure is taken to dominate in the dense shell. Flux conservation can be written as

$$
\frac{d\left(B_{t} / \rho r\right)}{d t}=0
$$

I assume that the outer shock has a radius $r_{s} \propto t^{\eta}$, so that the shock velocity is $v_{s} \propto t^{\eta-1}$. As in Bertschinger (1986), the compression in the radiative, thermal pressure-dominated region is described by a parameter $\lambda_{c}=\rho_{o} / \rho_{c}$, where $\rho_{o}$ is the ambient density and $\rho_{c}$ is the density at the base of the radiative region. For a case with no magnetic field and an isothermal shock front (as in Bertschinger 1986), $\lambda_{c} \propto \mathcal{M}^{-2} \propto v_{s}^{-2}$, where $\mathcal{M}$ is the shock Mach number. In the present case, the compression is limited by the magnetic field and $\lambda_{c} \propto \mathcal{M}_{a}^{-1} \propto v_{s}^{-1}$, where $\mathcal{M}_{a}$ is the Alfven Mach number. The thickness of the dense shell is $\sim \lambda_{c} r_{s}$, so the radial variable is changed to $\zeta$, defined by $r=r_{s}\left(1-\lambda_{c} \zeta\right)$. The radiative, thermally supported region is taken to be of negligible thickness, which should be a good approximation. Dimensionless variables for the physical variables can be defined by

$$
v=\frac{r_{s}}{t}\left(\eta-\lambda_{c} W_{o}\right), \quad \rho=\rho_{o} \lambda_{c}^{-1} E_{o}, \quad \frac{B_{t}^{2}}{8 \pi}=\rho_{o}\left(\frac{r_{s}}{t}\right)^{2}\left(1-\lambda_{c}\right) P_{o}
$$

The boundary conditions at $\zeta=0$ are $W_{o}=\eta, E_{o}=1$, and $P_{o}=\eta^{2}$.

The main assumption made here is that the shell thickness is much less than its radius, so that $\lambda_{c}$ can be regarded as a small parameter. Substitution of eqs. (6) into eqs. (4) and (5) yields

$$
\begin{gathered}
x \frac{d \ln E_{o}}{d \zeta}+\frac{d W_{o}}{d \zeta}+3 \eta-1=0, \\
\frac{d P_{o}}{d \zeta}+\eta(1-\eta) E_{o}=0 \\
x\left(\frac{d \ln P_{o}}{d \zeta}-2 \frac{d \ln E_{o}}{d \zeta}\right)-2 \eta=0
\end{gathered}
$$

where

$$
x=W_{o}-\zeta
$$

describes the gas velocity relative to a frame moving with the shell. The magnetic tension term does not appear in eq. (8) because it is higher order in $\lambda_{c}$. This is consistent with the numerical results of Slavin \& Cox (1992), who found that inclusion of this term did not substantially change their results. Integration of eqs. (7)-(10) can be initiated at $\zeta=0$ and carried to the inner boundary, where $x=0$. Results for $\eta=0.3$ are shown in Fig. 1. In contrast to the non-magnetic, adiabatic case where the density is constant over much of the shell and becomes large at the inner 
boundary (Bertschinger 1986), here the density decreases in from the shock front and drops at the inner boundary.

In their numerical calculations, Slavin \& Cox (1992) prefer the relation $d\left(B_{t} / \rho\right) / d t=0$ to that given in eq. (5) in order to give a lower bound on the magnetic effects. In this case, the flow in the shell is isentropic with $\gamma=2$ and the constant term drops out of eq. (9). The equations admit an analytic solution:

$$
\begin{gathered}
E_{o}=1-\frac{1-\eta}{2 \eta} \zeta, \quad P_{o}=\eta^{2}\left(1-\frac{1-\eta}{2 \eta} \zeta\right)^{2} \\
W_{o}=\frac{3 \eta^{2}}{1-\eta}+\left(1-\frac{3}{2} \eta\right) \zeta-\frac{2 \eta^{2}(4 \eta-1)}{(1-\eta)[2 \eta-(1-\eta) \zeta]}
\end{gathered}
$$

The inner boundary occurs at

$$
\zeta_{i}=\frac{2 \eta}{1-\eta}\left[1-\left(\frac{4 \eta-1}{3 \eta}\right)^{1 / 2}\right] .
$$

Results for this case for $\eta=0.3$ are shown in Fig. 2. The inner edge of the shell is at $\zeta_{i}=0.4531$. The density contrast across the shell is smaller than in the previous case, which brings the results closer to the non-magnetic case, as expected. The results compare well to the numerical computations of Slavin \& Cox (1992, fig. 1) during the time that the shell thickness is small compared to the radius.

Because there are approximations made in describing a 2-dimensional situation by a 1-dimensional flow, it is instructive to consider the 1-dimensional case of cylindrical expansion into a uniform magnetic field. The expansion is perpendicular to the field direction. The mass conservation equation is now

$$
\frac{d \rho}{d t}+\frac{\rho}{r} \frac{\partial r v}{\partial r}=0
$$

so that eq. (7) becomes

$$
x \frac{d \ln E_{o}}{d \zeta}+\frac{d W_{o}}{d \zeta}+2 \eta-1=0 .
$$

Now, mass and magnetic flux conservation imply that $B / \rho$ is conserved exactly. The flow in the shell is again isentropic so that $E_{o}$ and $P_{o}$ are described by eqs. (11). The velocity variable is given by

$$
W_{o}=\frac{2 \eta^{2}}{1-\eta}+(1-\eta) \zeta-\frac{2 \eta^{2}(3 \eta-1)}{(1-\eta)[2 \eta-(1-\eta) \zeta]} .
$$

and the inner boundary occurs at

$$
\zeta_{i}=\frac{2 \eta}{1-\eta}\left[1-\left(\frac{3 \eta-1}{2 \eta}\right)^{1 / 2}\right] .
$$

For cylindrical expansion, the case of momentum conserving expansion is $\eta=1 / 3$, so that now $\eta \geq 1 / 3$. The solution is very close to the one illustrated in Fig. 2. 


\subsection{Interaction with Clumps}

The supernova remnant becomes radiative in the interclump medium at a relatively small radius ( $\sim 5 \mathrm{pc}$ ) and interaction of the cool shell with a molecular clump becomes a matter of interest. This is the phase of evolution that is most likely to be observed because it lasts longer than the early adiabatic phase, and the observations of remnants discussed in the next section imply that the remnants are in the radiative phase. In the initial interaction, the radiative shell drives a dense slab into the clump. The slab is bounded by shock waves in the clump and in the radiative shell. The equations describing the evolution of the position $x$, surface density $\sigma$, and velocity $v$ of the slab are

$$
\begin{gathered}
\frac{d x}{d t}=v \\
\frac{d \sigma}{d t}=\rho_{r s}\left(v_{r s}-v\right)+\rho_{c l} v \\
\frac{d v}{d t}=\frac{\rho_{r s}\left(v_{r s}-v\right)^{2}-\rho_{c l} v^{2}}{\sigma}
\end{gathered}
$$

where $\rho_{r s}$ and $v_{r s}$ are the density and velocity in the radiative shell. Provided the thickness of the radiative shell is sufficiently small, $v_{r s}$ can be regarded as constant (see $\S 3.2$ ). However, the density $\rho_{r s}$ can vary with position in the shell.

The case of constant density $\rho_{r s}$ is particularly simple. The slab then moves at a constant velocity

$$
v=v_{r s}\left[1+\left(\frac{\rho_{c l}}{\rho_{r s}}\right)^{1 / 2}\right]^{-1} .
$$

The ram pressures generated by the two shock fronts are equal to each other. The ratio of the ram pressure in the slab to that at the front of the radiative shell is

$$
\frac{\rho_{c l} v^{2}}{\rho_{o} v_{r s}^{2}}=\frac{\rho_{c l}}{\rho_{o}}\left[1+\left(\frac{\rho_{c l}}{\rho_{r s}}\right)^{1 / 2}\right]^{-2} .
$$

The effect of the magnetic field is to limit $\rho_{r s}$ to somewhat below $\rho_{c l}$ so that the pressure ratio may be $\sim 10-100$. While the shock fronts are in the dense gas, the ratio of the swept up column density of clump gas to that from the radiative shell is simply $\left(\rho_{c l} / \rho_{r s}\right)^{1 / 2}$. If the quantity $\sigma_{c l} \rho_{r s}^{1 / 2} / \sigma_{r s} \rho_{c l}^{1 / 2}$ is $<1$, the shock front breaks out of the clump first, but if it is $>1$, the shock front breaks out of the radiative shell first. Here, $\sigma_{c l}$ and $\sigma_{r s}$ are the total column density through the clump and the radiative shell, respectively. If the shock front breaks out of the clump first, then the shocked clump is initially accelerated by the continued impact of the radiative shell.

For a more general density structure, it is useful to change to dimensionless variables. The position of the slab in the radiative shell is given by the value of $\zeta$ at the slab $\zeta_{s}$ and the radiative shell density can be described by $E_{o}(\zeta)$ as derived in $\S 3.2$. In addition, I define

$$
y=\frac{t}{\lambda_{c} \tau}, \quad U_{o}=\frac{v}{v_{s h}}, \text { and } \Omega=\frac{\sigma}{\rho_{s o} v_{r s} \lambda_{c} \tau},
$$


where $\tau=\eta r_{s} / v_{r s}$ is the age of the remnant. The substitution of these variables into eqs. (18)-(20) yields

$$
\begin{gathered}
\frac{d \zeta_{s}}{d y}=\left(1-U_{o}\right) \eta \\
\frac{d \Omega}{d y}=E_{o}\left(\zeta_{s}\right)\left(1-U_{o}\right)+\alpha U_{o} \\
\frac{d U_{o}}{d y}=\frac{E_{o}\left(1-U_{o}\right)^{2}-\alpha U_{o}^{2}}{\Omega}
\end{gathered}
$$

where $\alpha \equiv \rho_{c l} / \rho_{\text {so. }}$. Results for the case $E_{o}(\zeta)$ given by eq. (11) are shown in fig. 3. The slab receives an impulse from the initial dense part of the radiative shell that carries it forward even when the driving density in the shell drops. The slab decelerates, so that the forward pressure is greater than that at the shock front in the radiative shell, given by $\sigma_{r s} v_{r s}$, where $\sigma_{r s}$ is the mass column density through the radiative shell.

For the larger molecular clumps, the shock front breaks out of the rear of the radiative shell while the forward shock is in the molecular clump. Whatever the density structure in the radiative shell, the shell imparts a definite momentum to the motion of the slab. Initially, the interior pressure of the supernova remnant is small compared to the ram pressure of the slab, and the evolution of its position, $x$, can be described by

$$
\left(\sigma_{o}+x \rho_{c l}\right) \frac{d x}{d t}=\sigma_{r s} v_{r s} .
$$

where $\sigma_{o}$ is the column density in the slab at the end of the double shock phase. Once the swept-up column density becomes $\gg \sigma_{o}$, the position of the slab is

$$
x \approx\left(\frac{2 \sigma_{r s} v_{r s} t}{\rho_{c l}}\right)^{1 / 2}
$$

which describes the "snowplow" evolution in one dimension. The time $t$ here is initiated at the end of the double shock phase. If the remnant is well into the radiative phase, $\sigma_{r s} \approx \rho_{o} R / 3$. The time it takes the slab to traverse a distance $x$ can be written as

$$
\frac{t}{\tau}=\frac{3}{2 \eta}\left(\frac{\rho_{c l}}{\rho_{o}}\right)\left(\frac{x}{R}\right)^{2}
$$

where $\tau$ is the age of the remnant and $t<\tau$ has been assumed in deriving this expression. For the range of clump sizes expected in a molecular cloud, the small ones can be crushed soon after being hit by the radiative shell $(t<\tau)$, while the large ones continue to be shocked after they are left inside the remnant.

The approximate expression for the slab position (eq. [28]) can be used to find the surface density: $\sigma_{o}=x \rho_{c l} \approx\left(2 \sigma_{r s} v_{r s} \rho_{c l} t\right)^{1 / 2}$. The variables in eq. (23) can then be used to find an expression for $\Omega$ that should be approached at late times: $\Omega=(2 \alpha y / 3 \eta)^{1 / 2}$, where $\alpha \equiv \rho_{c l} / \rho_{s o}$. Fig. 3 shows that this provides a good representation of the column density. 


\subsection{The Relativistic Particle Component}

Consideration of relativistic electrons and protons in the supernova remnant is important for radio synchrotron radiation and for high-energy $\gamma$-ray emission. I will assume that the relativistic particles are accelerated in the shock front, although a central pulsar is another potential source of relativistic particles. I examine the case of propagation perpendicular to the magnetic field and assume that the relativistic particles are adiabatically compressed and maintain isotropic pitch angles. The gyroradii of the particles is smaller than the cooling length and the thickness of the cool shell, so that the adiabatic approximation is adequate.

The efficiency of production of the relativistic particles is not known, so I examine two simple cases. In the first (case I), a constant fraction of the flux of particles entering the shock front is injected into the relativistic particle population. The pressure of relativistic particles in the immediate postshock region is then $p_{r s}=\beta_{1} \rho_{o}$. In the second case (case II), the particle acceleration leads to a postshock pressure in relativistic particles that is proportional to the total postshock ram pressure so that $p_{r s}=\beta_{2} \rho_{o} v_{s}^{2}$. In both cases, the postshock evolution is adiabatic with $\gamma_{r}=4 / 3$. Assuming that the relativistic particle pressure does not become important in the cooling region, the relativistic pressure at the base of the radiative cooling region is $p_{r c}=p_{r s} /\left(4 \lambda_{c}\right)^{4 / 3}$.

The further evolution of the relativistic particle pressure, $p_{r}$, is then described by

$$
\frac{d p_{r}}{d t}-\frac{4 p_{r}}{3 \rho} \frac{d \rho}{d t}=0
$$

The solution for $p_{r}$ in the cool shell can be found from the solution for the shell in $\S 3.2$ provided that $p_{r}$ does not become an important contributor to the total pressure. The dimensionless variable for the relativistic pressure is defined by $p_{r}=p_{r c} P_{r}$ and eq. (30) becomes

$$
\begin{gathered}
\frac{d \ln P_{r}}{d \zeta}-\frac{4}{3} \frac{d \ln E_{o}}{d \zeta}=0 \quad(\text { case I }) \\
x\left(\frac{d \ln P_{r}}{d \zeta}-\frac{4}{3} \frac{d \ln E_{o}}{d \zeta}\right)-2(1-\eta)=0 \quad(\text { case II). }
\end{gathered}
$$

Case I is particularly simple because the relativistic fluid in the shell is isentropic so that

$P_{r}=E_{o}^{4 / 3}$. The case of $B_{t} \propto \rho$ (see eq. [11]) gives $P_{r}=[1-(1-\eta) \zeta / 2 \eta]^{4 / 3}$. In case II, the higher relativistic pressure generated at earlier times yields a high pressure in the inner part of the shell. The relativistic pressure comes to dominate inside the shell. We do not have a detailed theory that allows the properties of the relativistic particles to be calculated. The subject is deferred to $\S 5$, where the relevant observations are discussed. The conclusion of that discussion is that Case I is the relevant one for the observed remnants. 


\section{PROPERTIES OF OBSERVED REMNANTS}

A number of remnants are likely candidates for interaction with molecular clouds. Frail et al. (1996) list 8 remnants that show evidence for compact regions of $\mathrm{OH}$ maser emission. Here, I concentrate on the three remnants W44, IC 443, and 3C391, which have the most detailed observations. I use a distance of $3 \mathrm{kpc}$ to W44 (Wolszczan, Cordes, \& Dewey 1991), $1.5 \mathrm{kpc}$ to IC 443 (Fesen \& Kirshner 1980), and $9 \mathrm{kpc}$ to 3C391 (Reynolds \& Moffett 1993). The uncertainty in these estimates is $\sim 20-25 \%$, although the distance to W44 is probably more accurate because of the presence of a pulsar. In all 3 cases, the remnant radius is $\gtrsim 6$ pc so that the expansion in the interclump medium of a molecular cloud is expected to have entered the radiative phase (see $\S 3.1$ ). The general framework for understanding these objects is thus expected to be a radiative shell interacting with a clumpy molecular gas, unless the supernova surroundings have been modified by the progenitor evolution. The aim of this section is to examine the observational material on the remnants to check for consistency with this framework.

\subsection{W44}

Wootten (1977) identified W44 as a supernova remnant that is associated with molecular gas. These observations did show CO apparently in the vicinity of the remnant, although they did not conclusively show an interaction (DeNoyer 1983). However, Claussen et al. (1997) have found compact $\mathrm{OH}$ masers in the direction of the remnant (first discovered by Goss \& Robinson 1968) and Seta et al. (1998) have recently found evidence for CO clouds that are interacting with the remnant. The remnant is elongated, with radii of $11 \times 15 \mathrm{pc}$; because the present models are spherically symmetric, I take 13 pc for the radius.

The filled-center, thermal X-ray emission observed from a number of supernova remnants has led to a model involving the evaporation of clumps in the postshock region (White \& Long 1991). The evaporative model of Rho et al. (1994) for the X-ray emission from W44 has an intercloud ambient density of $n_{o}=0.09-0.26 \mathrm{~cm}^{-3}$, which is low for a molecular cloud, and the remnant is in an adiabatic phase. However, the multiwavelength observations of this remnant can be used to argue for a different type of model. Koo \& Heiles (1995) briefly discussed a radiative model for this remnant because they found an $\sim 350 M_{\odot}$ HI shell moving at $V_{s}=150 \mathrm{~km} \mathrm{~s}^{-1}$ that might be identified with the cool shell expected in the radiative phase. They also noted that the age of a radiative remnant, $0.3 R / V_{s}=29,000$ years where $R=15 \mathrm{pc}$ is the remnant radius, is in approximate agreement with the spindown age of the pulsar, PSR $1853+01$, that is inside the remnant. In the model of Rho et al. (1994), the age of the remnant is 6,000-7,500 years, which would require that the pulsar be born with a period close to its present period of $0.267 \mathrm{~s}$. However, Koo \& Heiles (1995) did not advocate the radiative model for W44, because it is not compatible with the filled-center X-ray emission.

Harrus et al. (1997) preferred the radiative model because the age would agree with that of 
the pulsar if it were born with a period of $10 \mathrm{msec}$. By computing hydrodynamic models, they found that the pulsar age and the mean remnant radius $(13 \mathrm{pc})$ could be fitted by models with $E_{o}=(0.7-0.9) \times 10^{51} \mathrm{ergs}$ and $n_{o}=(3-4) \mathrm{cm}^{-3}$. In these models, the shell velocity was $V_{s} \sim 120 \mathrm{~km} \mathrm{~s}^{-1}$, somewhat less than the $150 \mathrm{~km} \mathrm{~s}^{-1}$ found by Koo \& Heiles (1995). Alternatively, the relationship between $E_{o}, V_{s}, R$, and $n_{o}$ for radiative supernova remnants found by Chevalier (1974; eq. [46]) yields $n_{o}=4.7 \mathrm{~cm}^{-3}$ for $E_{o}=1 \times 10^{51} \mathrm{ergs}, V_{s}=150 \mathrm{~km} \mathrm{~s}^{-1}$, and $R=13 \mathrm{pc}$. Similar results can be obtained from the computations of Cioffi et al. (1988). Harrus et al. (1997) examined the X-ray properties of their radiative models and found that they could fit the centrally concentrated emission because of the effect of absorption. However, the models did show a greater temperature variation than that indicated by the observations.

Other observations support the view that W44 is in a radiative phase. Although optical observations are inhibited by dust obscuration, Giacani et al. (1997) have found H $\alpha$ and [SII] emission that covers the region of the radio remnant and, in places, shows a detailed correspondence with the radio structure. This correspondence is commonly found for radiative shock fronts. The range of $\mathrm{H} \alpha$ surface brightness found by Giacani et al. (1997) is $1 \times 10^{-17}-1 \times 10^{-16}$ ergs $\mathrm{cm}^{-2} \mathrm{~s}^{-1} \operatorname{arcsec}^{-2}$. Rho et al. (1994) estimate the reddening to W44 to be $E(B-V)=2.94$, which leads to an extinction $A_{\lambda} \approx 1.5 E(B-V)=4.4$ at the wavelength of $\mathrm{H} \alpha$. The extinction corrected $\mathrm{H} \alpha$ surface brightness is then $6 \times 10^{-16}-6 \times 10^{-15} \mathrm{ergs} \mathrm{cm}^{-2} \mathrm{~s}^{-1} \operatorname{arcsec}^{-2}$. In the radiative shock models of Shull \& McKee (1979), the $\mathrm{H} \beta$ surface brightness of a $130 \mathrm{~km} \mathrm{~s}^{-1}$ shock

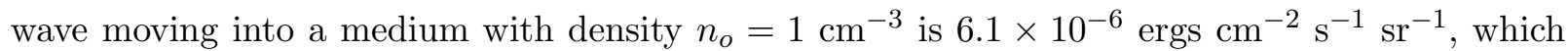
translates into an $\mathrm{H} \alpha$ surface brightness of $1.7 \times 10^{-15} \mathrm{ergs} \mathrm{cm}^{-2} \mathrm{~s}^{-1} \operatorname{arcsec}^{-2}$ for $n_{o}=4 \mathrm{~cm}^{-3}$. The surface brightness can be increased by projection effects when the shock front is moving close to perpendicular to the line of sight. The observed $\mathrm{H} \alpha$ surface brightness is thus consistent with the radiative model.

Another observational constraint is the [OI] $63 \mu \mathrm{m}$ line emission from the remnant. Reach \& Rho (1996) estimate that the observed luminosity in the line is $\sim 10^{3} L_{\odot}=4 \times 10^{36} \mathrm{erg} \mathrm{s}^{-1}$. They suggest that the remnant is in the adiabatic phase because this line is an important source of energy loss for the shock front and the loss rate times the age corresponds to the loss of a fraction of $10^{51}$ ergs. This claim can be checked by comparison with radiative shock models. Hollenbach \& McKee (1989) have calculated the emission from radiative shock fronts for velocities in the range $v_{s}=30-150 \mathrm{~km} \mathrm{~s}^{-1}$ and $n_{o}=10^{3}-10^{6} \mathrm{~cm}^{-3}$ and they find that the [OI] $63 \mu \mathrm{m}$ surface brightness is $10^{-6} n_{o} v_{2} \operatorname{ergs~} \mathrm{cm}^{-2} \mathrm{~s}^{-1} \mathrm{sr}^{-1}$, where $v_{2}$ is the shock velocity in units of $100 \mathrm{~km} \mathrm{~s}^{-1}$, for $n_{o} v_{2} \lesssim 10^{5} \mathrm{~cm}^{-2} \mathrm{~s}^{-1}$. The reason that the line surface brightness is proportional to the shock particle flux is that the line is responsible for most of the gas cooling below a temperature of 5,000 K. The results of Raymond (1979) show that this relation can be extended down at least to $n_{o}$ of order unity. The luminosity in the line is $1.5 \times 10^{35} n_{o} v_{2} R_{1}^{2} \mathrm{erg} \mathrm{s}^{-1}$, where $R_{1}$ is the radius in units of $10 \mathrm{pc}$. This is a small fraction of the total radiative loss. For $v_{2}=1.5, n_{o}=4.7 \mathrm{~cm}^{-3}$, and $R=13 \mathrm{pc}$, the expected [OI] $63 \mu \mathrm{m}$ luminosity is $2 \times 10^{36} \mathrm{erg} \mathrm{s}^{-1}$ The observed luminosity is a factor of 2 higher than that expected in the radiative model, but this is within the observational 
uncertainty considering that only a portion of the remnant has been observed (Reach \& Rho 1996). The observed [OI] $63 \mu \mathrm{m}$ luminosity thus provides support for the radiative model. The peak surface brightness in the [OI] $63 \mu \mathrm{m}$ line is $\sim 10^{-3} \mathrm{ergs} \mathrm{cm}^{-2} \mathrm{~s}^{-1} \mathrm{sr}^{-1}$ (Reach \& Rho 1996), which requires either shock motion into a dense region or projection effects for an edge-on shock front.

The observations of W44 can thus be modeled as a $10^{51}$ erg explosion in a medium with density $4-5 \mathrm{~cm}^{-3}$ and current shock radius of $13 \mathrm{pc}$. The shock front is radiative and the ambient density is at the low end of the range expected for the interclump density of a molecular cloud. The interaction with molecular clumps is probably best observed in the $\mathrm{OH}$ maser emission (Claussen et al. 1997), which is likely to come from a shock front in molecular gas (Elitzur 1976). An important aspect of these observations is that the Zeeman effect allows the measurement of the magnetic field strength along the line of sight, $B_{l}$. Claussen et al. (1997) find $B_{l} \sim 3 \times 10^{-4}$ $\mathrm{G}$ in W44, which is close to the peak field strength expected in the cool shell $\left(2.5 \times 10^{-4} \mathrm{G}\right.$ for $n_{1}=0.47$ and $v_{2}=1.5$, see $\S 3.1$ ). As discussed in $\S 3.3$, the pressure in the cooling region of a clump shock can be higher than that in the radiative shell and the magnetic field strength is correspondingly increased.

\subsection{IC 443}

IC 443 has strong molecular emission that makes it the clearest case of supernova interaction with a molecular cloud. The cloud appears to be in front of the remnant (Cornett, Chin, \& Knapp 1977), so that the interaction is primarily approaching us. The molecular cloud found by Cornett et al. (1977) has a mass of only $\lesssim 10^{4} M_{\odot}$. This is low for a molecular cloud and, as argued in $\S 2$, is the type of cloud that is more likely to show the interaction of a supernova remnant with the molecular material. Braun \& Strom (1986) noted that the IC 443 complex could be described by 3 overlapping shells (A, B, and C) with different radii of curvature. They attributed this structure to the expansion of the remnant into shell structures that were created by the actions of stellar winds. X-ray observations of shell $\mathrm{C}$ now show that it is a separate, older supernova remnant in front of IC 443 (Asaoka \& Aschenbach 1994). For shells A and B, I take the point of view that they are one remnant; shell A is expanding into the interclump medium of a molecular cloud and shell B is the breakout into the low density surroundings. The breakout morphology is most clearly shown by images at X-ray (Asaoka \& Aschenbach 1994, Fig. 2) and radio (e.g., Claussen et al. 1997) wavelengths. I estimate the radius of shell A to be $7.4 \mathrm{pc}$.

Shell A comprises about half of the solid angle subtended by the remnant and includes the bright optical filaments to the NE of the remnant. Fesen \& Kirshner (1980) found that the spectra of these filaments imply an electron density for the [SII] emitting region of $\lesssim 100$ to $500 \mathrm{~cm}^{-3}$ and shock velocities in the range $65-100 \mathrm{~km} \mathrm{~s}^{-1}$. If the magnetic field does not limit the compression to $\sim 10^{4} \mathrm{~K}$, the preshock density implied by the higher density filaments is $10-20 \mathrm{~cm}^{-3}$. The filaments with a lower density may have their compression limited by the magnetic field. The 
physical parameters are in approximate agreement with those derived from HI observation. For an ambient density of $15 \mathrm{~cm}^{-3}$ and a radius of $7.4 \mathrm{pc}$, the total swept-up mass is $1000 M_{\odot}$ for a half-sphere. The HI observations of Giovanelli \& Haynes (1979) suggest that there is $\sim 1000 M_{\odot}$ of shocked HI in the shell A part of the remnant, with velocities up to $\sim 110 \mathrm{~km} \mathrm{~s}^{-1}$. The application of eq. (46) of Chevalier (1974) for a spherical remnant yields $E=4 \times 10^{50}$ ergs for $v_{s}=100 \mathrm{~km} \mathrm{~s}^{-1}, R=7.4 \mathrm{pc}$, and $n_{o}=15 \mathrm{~cm}^{-3}$. This energy probably underestimates the initial total energy because the breakout into the low density region decreases the pressure driving the dense shell.

As for $\mathrm{W} 44$, the radiative shell should give rise to [OI] $63 \mu \mathrm{m}$ emission. This line has been observed from IC 443, but only from a small region with a molecular clump (Burton et al. 1990). For $v_{s}=100 \mathrm{~km} \mathrm{~s}^{-1}$ and $n_{o}=15 \mathrm{~cm}^{-3}$, the [OI] $63 \mu \mathrm{m}$ line luminosity from the half shell should be $\sim 1 \times 10^{36} \mathrm{erg} \mathrm{s}^{-1}$. Mufson et al. (1986) found that the luminosity of the remnant in the IRAS $60 \mu \mathrm{m}$ band is $\sim 1 \times 10^{37} \mathrm{erg} \mathrm{s}^{-1}$. About $10 \%$ of this band luminosity may be in the [OI] line. This line provides an excellent means to investigate the radiative shell because it is relatively unaffected by dust absorption in the the cloud that covers the central part of the remnant.

The radiative shell model provides an adequate description of the observations of shell A. The model implies an age $t \approx 0.3 R / v_{s}=30,000 \mathrm{yr}$ for $r=7.4 \mathrm{pc}$ and $v_{s}=100 \mathrm{~km} \mathrm{~s}^{-1}$. This age is greater than some other estimates based on X-ray observations. The brightest region in X-rays is to the $\mathrm{N}$ and has a temperature $\sim 10^{7} \mathrm{~K}$ (e.g., Petre et al. 1988), which corresponds to a shock velocity of $\sim 800 \mathrm{~km} \mathrm{~s}^{-1}$ if it is in an immediate postshock region. In the current model, the shock reaches the beginning of the cooling phase at $v_{s} \approx 600 \mathrm{~km} \mathrm{~s}^{-1}$ when the radius is $4 \mathrm{pc}$ and there is hotter gas in the interior. The action of heat conduction may distribute the high interior heat content so that the temperature is $\sim 10^{7} \mathrm{~K}$ despite the expansion of the radiative shell. Petre et al. (1988) estimate the X-ray emitting mass to be only $\sim 10 M_{\odot}$. If this model cannot explain the high temperature, a model involving interaction with a pre-existing shell may be necessary, so that the shock front has been moving rapidly until recently.

Recently, Wang et al. (1992) found evidence for gas with a temperature $\gtrsim 10^{8} \mathrm{~K}$ and concluded that the remnant's age is $\sim 1,000$ years. This age has been assumed in other X-ray studies (Asaoka \& Aschenbach 1994; Keohane et al. 1997). However, Keohane et al. (1997) present $A S C A$ observations of the region and concluded that synchrotron emission provides a more consistent explanation of the hard X-ray emission, which removes the main argument for the small age. Most of the hard X-ray emission comes from a centrally concentrated source in the southern part of the remnant (Keohane et al. 1997). This region also has a relatively flat radio spectral index, $\alpha<0.24$, where flux $\propto \nu^{-\alpha}$ (Green 1986). These properties point to the presence of a pulsar-driven nebula. Keohane et al. (1997) argue against this interpretation for a number of reasons, the strongest of which is that the pulsar would have to have a velocity $\gtrsim 5,000 \mathrm{~km} \mathrm{~s}^{-1}$ to reach its position near the edge of the remnant. With the age advocated here, the required velocity drops to $\lesssim 200 \mathrm{~km} \mathrm{~s}^{-1}$ and the problem disappears; IC 443 probably contains a low luminosity pulsar nebula that has little influence on the shock wave interactions. Keohane et al. (1997) 
attribute the emission to energetic electrons accelerated by the shock front at edge of the remnant and find another extended hard X-ray region at the eastern edge of the remnant. A shock with velocity $\sim 100 \mathrm{~km} \mathrm{~s}^{-1}$, as in the present model, is not a promising place to accelerate electrons to energies $\sim 10 \mathrm{TeV}$, but this region is relatively faint and needs to be observed in more detail.

In a restricted region across the central, eastern part of IC 443, a rich spectrum of molecular emission has been observed (van Dishoeck et al. 1993; Richter at al. 1995b, and references therein). A set of spectroscopically distinct CO clumps (labelled A-G) has been listed by DeNoyer (1979b) and Huang, Dickman, \& Snell (1986). These clumps, with sizes $\sim 1$ pc, have masses of $3.9-41.6 M_{\odot}$ as deduced from ${ }^{12} \mathrm{CO}$ lines (Dickman et al. 1992). The corresponding densities, $n_{H} \lesssim 500 \mathrm{~cm}^{-3}$, are low but should be regarded as lower limits because the ${ }^{12} \mathrm{CO}$ emission is assumed to be optically thin. Analysis of absorption in one place yielded a preshock density of $n\left(\mathrm{H}_{2}\right) \approx 3,000 \mathrm{~cm}^{-3}$ (van Dishoeck et al. 1993). The properties of these clumps are consistent with those seen in quiescent molecular clouds (see $\S 2$ ). Especially high spatial resolution is possible in the $\mathrm{H}_{2}$ infrared lines (Richter et al. 1995b), which show clumps down to a scale of $1^{\prime \prime}$ $\left(2 \times 10^{16} \mathrm{~cm}=0.007 \mathrm{pc}\right.$ at a distance of $\left.1.5 \mathrm{kpc}\right)$ but not smaller. Richter et al. (1995b) note that the emission has a knotty appearance that is unlike the filamentary appearance of the optical emission in IC 443 and the $\mathrm{H}_{2}$ emission in the Cygnus Loop. This type of structure is consistent with the fractal structure of molecular clumps deduced by Falgarone \& Phillips (1991).

The interpretation of the molecular line strengths is still controversial, but the emission is most consistent with that from a partially dissociating $J$ (jump)-type shock (Burton et al. 1990; van Dishoeck et al. 1993; Richter at al. 1995a,b). The required shock velocity, $25-30 \mathrm{~km} \mathrm{~s}^{-1}$, is consistent with the velocities observed in the strongest emission. The dissociated $\mathrm{H}_{2}$ can be observed as a high column density of shocked HI at the positions of the clumps (Braun \& Strom 1986). For $v_{c}=25-30 \mathrm{~km} \mathrm{~s}^{-1}$ and $v_{r s}=100 \mathrm{~km} \mathrm{~s}^{-1}, \rho_{c l} / \rho_{r s}$ must be in the range $5.4-9.0$ according to eq. (21). The density ratio can vary significantly with relatively little effect on the shock velocity. The clump shock velocity is consistent with $n_{r s}=500 \mathrm{~cm}^{-3}$ and $n_{c l}=3000 \mathrm{~cm}^{-3}$, which are plausible values. The ratio of postshock pressure in the clump to that in the interclump medium (with $n_{o}=15 \mathrm{~cm}^{-3}$ ) is 18 , which is different from the ram pressure equilibrium typically found in cloud interactions in adiabatic supernova remnants (McKee \& Cowie 1975). Moorhouse et al. (1991) found a high pressure in IC 443 from $\mathrm{H}_{2}$ observations and proposed an explanation similar to that given here. I have shown how the required radiative shell follows naturally from the global properties of the supernova remnant.

The column density through the radiative shell is $N_{H} \approx n_{o} R / 3 \approx 10^{20} \mathrm{~cm}^{-2}$. For $n_{c l}=3000$ $\mathrm{cm}^{-3}$, the column density through the clumps is $10^{22} \ell_{\mathrm{pc}} \mathrm{cm}^{-2}$, where $\ell_{\mathrm{pc}}$ is the path length through the clump in pc. The larger observed clumps are thus expected to be passed over by the radiative shell and left in the interior of the remnant (see $\S 3.3$ ). For clumps with a size $\lesssim 0.02 \mathrm{pc}$, the shock front breaks out of the clump first and there is the possibility of further acceleration by the radiative shell. The initial shock front through the clump may not dissociate molecules, which are then accelerated by the shell. Low column densities of high velocity molecular gas can 
be produced in this way. High velocity molecular gas has been observed in IC 443 at the edge of one of the clumps (Tauber et al. 1994).

\section{3. $\quad 3 \mathrm{C} 391$}

3C391 is another remnant that is likely to be interacting with a molecular cloud, based on the apparent breakout morphology at radio and X-ray wavelengths (Reynolds \& Moffett 1993; Rho \& Petre 1996) and on the detection of $\mathrm{OH}$ maser emission (Frail et al. 1996). At a distance of $9 \mathrm{kpc}$, the bright radio emission to the NW, which is presumably the cloud interaction, has a radius of curvature of $6 \mathrm{pc}$. This radius is just bigger than that of the transition to the radiative phase for an interclump density of $10 \mathrm{~cm}^{-3}$ (see $\S 3.1$ ). The dynamical status of the remnant thus depends on the exact interclump density, but if the remnant has made a transition to the radiative phase, the shock velocity may still be 100 's of $\mathrm{km} \mathrm{s}^{-1}$. The remnant has not been detected at optical wavelengths; Reynolds \& Moffett (1993) attribute this to the large absorption along the line of sight. The [OI] $63 \mu \mathrm{m}$ line is not much affected by absorption. It has been detected in two places in 3C391 and the whole remnant is inferred to have a line luminosity $\sim 10^{3} L_{\odot}=4 \times 10^{36} \mathrm{erg} \mathrm{s}^{-1}$. In the shock model for the line, the luminosity for a spherical remnant is $L_{[\mathrm{OI}]}=1.5 \times 10^{36} n_{1} v_{2} R_{1}^{2} \mathrm{erg} \mathrm{s}^{-1}$, where $R_{1}$ is the remnant radius in units of 10 pc. The line luminosity for $R_{1}=0.6$ and $v_{2}=3$ is thus $L_{[\mathrm{OI}]}=1.6 \times 10^{36} n_{1} \mathrm{erg} \mathrm{s}^{-1}$, which is in adequate agreement with the observations, considering that only part of the remnant was observed. Although the spectral resolution available to Reach \& Rho (1996) was poor, they found that the velocity centroid of the [OI] line was shifted by $\sim 400 \mathrm{~km} \mathrm{~s}^{-1}$ between the two places that they detected the line. This velocity difference is suggestive of the velocities in newly formed radiative shock fronts, which are faster than the shocks in molecular clumps.

Reach \& Rho (1998) have recently found that there is strong molecular emission from a shocked clump in the southern part of the remnant. They note that the CO line brightness implies a pressure $\sim 10^{2}$ higher than that expected for a $10^{51} \mathrm{erg}$ supernova. As discussed in $\S 3.3$, an overpressure of this order can be generated if a radiative shell interacts with a dense clump.

\subsection{X-ray Emission and Heat Conduction}

The X-ray observations of the 3 remnants discussed in $\S 4$ present the greatest challenge to the type of model proposed here. In the standard model for a radiative supernova remnant, there is hot gas left at the center. However, the hot gas has its highest density close to the shell and the temperature increases toward smaller radii (Chevalier 1974; Cioffi et al. 1988; Harrus et al. 1997). The result is the expectation of a shell X-ray source with a decreasing temperature toward larger radii. The observations of these remnants show centrally concentrated thermal X-ray emission with little temperature variation (Rho et al. 1994 on W44; Petre et al. 1988 on IC 443; Rho \& 
Petre 1996 on 3C391). Harrus et al. (1997) have attempted to solve this problem for W44 by suggesting that the shell emission is absorbed, leaving the centrally concentrated emission.

These remnants belong to a larger class of remnants that are characterized by their center-filled, thermal X-ray emission. Their X-ray characteristics have been used to advocate the evaporating cloud model of White \& Long (1991). In this model, the main supernova shock front propagates without losing energy through an intercloud medium. Embedded in this medium are dense clouds that are not accelerated by the passage of the blast wave. The clouds find themselves in a hot medium, which leads to evaporation of the cloud material by saturated heat conduction. White \& Long (1991) found that if the mass evaporation rate has a $t^{-1}$ time dependence, the flow can be described by a self-similar solution. If the evaporation time is short, the clouds are completely evaporated close to the shock front. If the evaporation time is very long, the blast wave is not affected by the clouds. But there is an intermediate regime in which the cloud evaporation adds mass to the center of the supernova remnant and can give a center-filled remnant. The remnants are taken to be in this regime and most of the X-ray emission comes from evaporated clump gas. Applications of this model lead to low values for the preshock intercloud $\mathrm{H}$ density: 0.09-0.26 $\mathrm{cm}^{-3}$ in W44 (Rho et al. 1994), $0.039 \mathrm{~cm}^{-3}$ in W28 (Long et al. 1991) which is another source of $\mathrm{OH}$ maser emission (Claussen et al. 1997), and $0.07-0.23 \mathrm{~cm}^{-3}$ in $3 \mathrm{C} 391$ (Rho \& Petre 1996).

In both the present model and the evaporative model, the remnants are interacting with a clumpy medium, but the nature of the interclump medium is very different in the two models. The discussion in this section provides strong arguments that the 3 remnants under consideration are interacting with a dense ambient medium and have developed radiative shells. There are 2 reasons why clump evaporation is unlikely to be an important process in the present model. First, evaporation leads to a higher interior density, which helps with modeling the X-ray structure. However, the cooling time is shortest in the interior, so that the radiative shell forms well inside of the outer shock front, as can be seen in the numerical calculations of Cowie, McKee, \& Ostriker (1981). This is incompatible with the observations. The other reason is that the estimated mass of X-ray emitting gas is much less than the estimated mass of the radiative shells. Clump evaporation may be taking place, but not at a level that is important for the evolution.

Although clump evaporation is probably not significant, heat conduction in the hot interior gas probably is significant. Harrus et al. (1997) found that their non-conduction models were unable to fit the isothermal nature of the X-ray emission from W44. Conduction in the interclump gas is plausible because stellar polarization observations of molecular clouds show that this gas has a significant uniform component to the magnetic field (see $\S 2$ ). Models for radiative remnants with heat conduction are presented by Cui \& Cox (1992). The conditions here are such that conduction does not impede formation of the radiative shell, but can operate in the hot interior. 


\section{NONTHERMAL PARTICLES AND THEIR EMISSION}

The presence of relativistic electrons in supernova remnants like those discussed in $\S 4$ has long been inferred from their radio emission. A recent development has been the likely detection of a number of supernova remnants in energetic $\gamma$-rays, including W44, IC 443, and W28 (Esposito et al. 1996). Possible sources of the emission are bremsstrahlung and inverse Compton radiation associated with energetic electrons and pion decays associated with energetic protons. These emission mechanisms have been extensively studied in the context of the supernova remnant emission (Sturner et al. 1997; Gaisser, Protheroe, \& Stanev 1998). Although these studies have treated the emission mechanisms in detail, their treatments of the supernova remnant structure are more rudimentary. For example, Sturner et al. (1997) present a model for IC 443 in which the interior density is constant at 4 times the ambient density of $10 \mathrm{~cm}^{-3}$ and the magnetic field is constant at 4 times the ambient value of $5 \mu \mathrm{G}$. They assume that particle acceleration terminates when a remnant enters the radiative phase, but have IC 443 as non-radiative at an age of 5,000 yrs. Here, I examine the nonthermal component in the context of the supernova remnant models developed in the previous section.

The nonthermal component is observed at the highest spatial resolution at radio wavelengths. A close correlation between the radio and optical structure has been found in IC 443 (Duin \& van der Laan 1975) and in W44 (Giacani et al. 1997). The optical structure in IC 443 and other radiative shock front regions is best explained as emission from a wavy sheet. The fact that the radio emission shows a similar structure implies that a major part of it is associated with a thin region near the radiative shock. Duin \& van der Laan (1975) attribute the emission to the compressed ambient magnetic field and cosmic ray electrons. Particle acceleration at the shock front can also be a factor (e.g., Blandford \& Cowie 1982).

Radiative shock fronts are also driven into the dense clump gas and the high pressure should result in a high magnetic field strength in the postshock region ( $\S 3.3)$. However, the clumps do not appear to be significantly enhanced sources of radio synchrotron emission. Figures 2 and 3 of Claussen et al. (1997) show that except for the sources OH D and OH E in W44, there is little correlation between the compact $\mathrm{OH}$ masers and the radio continuum emission in $\mathrm{W} 44$ and IC 443. In IC 443, the small region of $\mathrm{OH}$ masers does match up with a CO clump (Claussen et al. 1997), but the radio continuum emission shows little correlation with the CO clumps (Dickman et al. 1992). The absence of strong emission is not surprising because the shock fronts into the clumps are not ionizing shock fronts. Under these circumstances, Alfven waves are damped in the neutral gas and energetic particles may be able to escape from the shock region.

The conclusion is that the radio emission is from the compressed regions behind the radiative shock fronts in the interclump gas. A possible problem with this source is free-free absorption by compressed gas in the cooling, recombining region. Erickson \& Mahoney (1985) have pointed out the lack of free-free absorption in IC 443 down to $\sim 20 \mathrm{MHz}$, which may be a problem in a radiative shock model. At $10 \mathrm{MHz}$, absorption does appear to be present. I estimate the free-free 
optical depth from the intensity of $H \beta$ emission expected from a radiative shock front. Taking the $H \beta$ intensity from Raymond's (1979) shock models for a $100 \mathrm{~km} \mathrm{~s}^{-1}$ shock and the $H \beta$ emissivity coefficient from Osterbrock (1989), I find the free-free depth at $T \approx 8,000 \mathrm{~K}$ to be

$$
\tau_{f f} \approx 0.01 \nu_{8}^{-2} n_{1}
$$

For IC 443, the cooling region is just becoming optically thick at $10-20 \mathrm{MHz}$, which is roughly compatible with the observations. The $\mathrm{H} \beta$ intensity observed by Fesen \& Kirshner (1980) is higher than that in the shock model by $\gtrsim 10$ in some bright filaments, which can be attributed to the fact that bright filaments are probably shock fronts viewed edge-on.

The observed radio flux from IC 443, 160 Jy at 1 GHz (Erickson \& Mahoney 1985), can be used to find the energy density of the relativistic electrons in the context of the radiative shell model. The emitting volume of the thin shell is $4 \pi R^{2} \Delta R \approx\left(4 \pi R^{3} / 3\right)\left(\rho_{o} / \rho_{s h}\right)$, where $\left(\rho_{o} / \rho_{s h}\right)=15 / 500$. With $R=7.4 \mathrm{pc}$, the emitting volume is $1.5 \times 10^{57} \mathrm{~cm}^{3}$. I take $B \sin \theta=2 \times 10^{-4} \mathrm{G}$ in the thin shell, so that the emitting electrons at $1 \mathrm{GHz}$ have energies $E \approx 0.56 \mathrm{GeV}$. The radio spectral index is observed to be $\alpha=0.36$ (Erickson \& Mahoney $1985)$, where $\alpha$ is defined by flux $\propto \nu^{-\alpha}$; this corresponds to an energy spectral index $\Gamma=1.72$ $\left(N(E) d E \propto E^{-\Gamma} d E\right)$. The basic parameters given here for the radiative shell of IC 443 are close to those given by Duin \& van der Laan (1975), and the radio flux can again be attributed to the compression and acceleration of ambient cosmic ray electrons in the dense shell. Blandford \& Cowie (1982) discussed particle acceleration in older remnants with radiative shock waves, like IC 443. They noted that very efficient cosmic ray acceleration at the shock front cannot be occurring, or the high compression in the radiative region of the shock front could not take place. However, in their model the ambient cosmic ray electrons are accelerated in the shock front by the first-order Fermi mechanism, which produces a $\Gamma=2$ energy spectrum. This is significantly steeper than the spectral index observed in IC 443. If the emitting electrons are compressed ambient cosmic rays, the spectral index may depend on that of the ambient cosmic rays. As a first approximation, I take the cosmic ray spectrum in the cloud to be the same as that in the Galaxy. Observations of molecular clouds with $C G R O$ indicate that the cosmic ray content of molecular clouds is similar to that in the rest of the interstellar medium (Digel et al. 1995, 1996). Taking into account the compression in the radiative shock front, the initial preshock electron energy range covered by the $30 \mathrm{MHz}-10 \mathrm{GHz}$ observations of IC 443 is $50-900 \mathrm{MeV}$. Longair (1994, p. 284) estimates $\Gamma=1.6$ over the energy range $10-100 \mathrm{MeV}$ and $\Gamma=1.8$ over $100 \mathrm{Mev}-1 \mathrm{GeV}$ for ambient, interstellar cosmic ray electrons.

In shock acceleration in the test particle limit (Blandford \& Ostriker 1978), an input spectrum with $\Gamma>2$ results in $\Gamma=2$ in the postshock region. If $\Gamma<2$ initially, the particle spectrum is preserved in the postshock region. The relatively flat ambient cosmic ray spectrum is thus preserved in the postshock region and can explain the flat radio synchrotron spectrum that is observed in IC 443. This spectrum would be difficult to understand if electrons were injected at the shock front. Longair (1994) attributes the flat spectrum of the ambient cosmic rays to Coulomb losses in the interstellar medium, which he estimates as $10 n \mathrm{eV} \mathrm{yr}^{-1}$ where $n$ in the gas 
density in $\mathrm{cm}^{-3}$. Coulomb losses in the dense shell of the supernova remnant are also possible. For particle energy $E=500 \mathrm{MeV}$ and $n=500 \mathrm{~cm}^{-3}$, the loss time is $E(d E / d t)^{-1}=10^{5} \mathrm{yr}$. This is longer than the estimated age of the remnant $\left(3 \times 10^{4} \mathrm{yr}\right)$, but is suffiently close that Coulomb losses in the shell could be a factor for some remnants.

The electron particle spectrum required to produce the observed flux from IC 443, given the above spectrum and volume, is $N(E)=N_{o} E^{-\Gamma}=3 \times 10^{-11} E^{-1.72}$ in cgs units. On the assumption that the particle pitch angles are isotropized during the postshock compression, the individual particles gain energy as $s^{1 / 3}$, where $s$ is the compression factor, and $N_{o} \propto s^{(2+\Gamma) / 3}$. From the theory of Blandford \& Ostriker (1978), the shock acceleration of the ambient cosmic rays leads to an increase in $N_{o}$ by a factor of 14 . The combination of these factors implies that the electron spectrum ahead of the shock front is $N(E)=2 \times 10^{-14} E^{-1.72}$. This spectrum compares adequately with the spectrum $N(E)=2 \times 10^{-14} E^{-1.6}$ given by Longair (1994) for ambient cosmic ray electrons up to $\sim 100 \mathrm{MeV}$. In the present model, the electron energy density in the dense shell is $\sim 1 \%$ of the magnetic energy density. I have neglected the structure of the radiative shell, which corresponds to Case I in $\S 3.4$, but this should not significantly affect the results. The present model for the radio emission resembles that of Blandford \& Cowie (1982) except for the difference in spectral index and in the overall picture. Blandford \& Cowie take the emission to be from crushed interstellar clouds, whereas here it is from the radiative shell in the interclump medium of a molecular cloud.

Further support for the molecular cloud model comes from polarization observations of the radio synchrotron emission. Kundu \& Velusamy (1972) found that both IC 443 and W44 show an ordered field direction and that the polarization in W44 is up to $20 \%$, which is unusually high for a supernova remnant. In the case of IC 443, the magnetic field direction deduced from the synchrotron emission lines up with that deduced from interstellar polarization in the surrounding cloud. The emission is thus consistent with the compressed magnetic field coming from the interclump component of a molecular cloud with a significant uniform magnetic field (see $\S 2$ ).

The relativistic electrons that give rise to synchrotron radiation can also give rise to bremsstrahlung radiation. The bremsstrahlung intensity for a power law electron spectrum is $1.0 \times 10^{-15} n N_{o} E^{-\Gamma} /(\Gamma-1) \mathrm{cm}^{-3} \mathrm{ergs}^{-1} \mathrm{~s}^{-1}$ (Longair 1994, p. 269). If electrons of an energy $E$ can be considered to give synchrotron radiation at a frequency $\nu_{s}$ and bremsstrahlung radiation at energy $E$, then the ratio of bremsstrahlung power to synchrotron power for those electrons is

$$
\frac{\left(\nu L_{\nu}\right)_{\mathrm{brems}}}{\left(\nu L_{\nu}\right)_{\mathrm{syn}}}=110\left(\frac{n}{500 \mathrm{~cm}^{-3}}\right)\left(\frac{B \sin \theta}{2 \times 10^{-4} \mathrm{G}}\right)^{-3 / 2}\left(\frac{\nu_{s}}{1 \mathrm{GHz}}\right)^{-1 / 2},
$$

where the bremsstrahlung emission is at photon energy $E$, the radio synchrotron emission is at frequency $\nu_{s}$, and $\Gamma=2$ has been assumed. A similar calculation for $\Gamma=1.72$ gives $\sim 150$ for the ratio when the reference values are used. For IC 443, an electron radiating at $1 \mathrm{GHz}$ has $E=0.56$ $\mathrm{GeV}$. The reference values for IC 443, used in eq. (34), then give the ratio of $\gamma$-ray power (at 0.56 $\mathrm{GeV}$ ) to radio synchrotron power (at $1 \mathrm{GHz}$ ) for the remnant. The observed value for the ratio 
is about 30 (Esposito et al. 1996; see fig. 11 of Sturner et al. 1997). The current model thus gives the correct ratio to order of magnitude. Sturner et al. (1997) also propose a bremsstrahlung model for the high energy $\gamma$-ray emission from IC 443 , but in the context of a different supernova remnant model (uniform $n=40 \mathrm{~cm}^{-3}$ and $B=2 \times 10^{-5} \mathrm{G}$ inside the remnant).

Other possibilities for the high energy $\gamma$-ray emission are inverse Compton emission and pion decays triggered by the collisions of relativistic protons with thermal gas. The importance of inverse Compton compared to bremsstrahlung depends on the ratio of the radiation energy density to the gas density. Gaisser et al. (1998) have discussed the radiation energy density in IC 443. For $n=500 \mathrm{~cm}^{-3}$ taken here, the inverse Compton mechanism is not a factor. The spectrum expected for pion decay $\gamma$-ray emission does not fit the observed $\gamma$-ray spectrum (Esposito et al. 1996; Sturner et al. 1997; Gaisser et al. 1998) so that bremsstrahlung emission must be a significant contributor to the emission in the present model. Pion decay emission may still make a contribution to the $\gamma$-rays, but a detailed calculation of the emission is beyond the scope of this paper. The present model does give a definite scenario (acceleration and compression of ambient cosmic rays) that can be examined.

The nonthermal emission from the other supernova remnants can be investigated from the same point of view. The radio spectral index of W44 is $\alpha=0.33$ (Kovalenko, Pynzar, \& Udal'tsov 1994), which can again be explained in the present model by the acceleration and compression of ambient cosmic ray electrons. Because of the flat spectral index, de Jager \& Mastichiadis (1997) suggest a model in which the electrons come from the pulsar associated with the remnant. The ability of the electrons to move into a shell with a tangential magnetic field is not clear. In the case of 3 C391, the spectral index is steeper, $\alpha \approx 0.55$ (Goss et al. 1979). One possibility is that this remnant has recently made the transition to being radiative in the interclump medium and there is an important contribution to the relativistic electrons from particles that have been injected into the shock acceleration process during the earlier phase.

The main point here is not to present definitive models for the remnants, but to give supernova remnant model that can be used in more detailed treatments of the various types of emission. The approximate results appear promising for more detailed calculations.

\section{DISCUSSION}

The aim of this paper has been to show that observations of a number of luminous supernova remnants can be interpreted in terms of interaction with a clumpy molecular cloud. The remnants become radiative in the interclump medium and the radiative shells then interact with molecular clumps. The magnetically supported radiative shells can be observed as cool HI shells and by the radiative shock emission. Because the extinction is frequently high to these remnants in dense regions, the study of infrared line emission can be especially fruitful. The [OI] $63 \mu \mathrm{m}$ line has already been found to be a powerful probe of these remnants. A prediction of this model is that 
the high-energy $\gamma$-ray emission detected from some of these remnants is from the radiative shell. This prediction can eventually be checked with experiments that have a higher spatial resolution than is currently possible, such as GLAST (Gamma-ray Large Area Space Telescope). A more detailed treatment of the expected emission spectrum for acceleration of ambient cosmic ray should also be carried out.

The interaction of the radiative shell with molecular clumps can give a number of features observed from these remnants, such as higher pressures than those in the radiative shell and the acceleration of molecules to relatively high velocities. Observations of molecular clouds have shown a great deal of small scale structure in the molecular material and this can be seen directly in $\mathrm{H}_{2}$ observations of the remnant IC 443 (Richter et al. 1995b). There may also be instabilities associated with the interaction. Numerical computations of the interaction of a radiative shell with a dense clump should be carried out to elucidate the hydrodynamic features of the interaction. These calculations will be helpful in the interpretation of detailed molecular shock wave observations.

The hot interiors of the remnants are a source of X-ray emission. The observations show that the emission is close to isothermal, which suggests the action of heat conduction. However the evaporation of clumps does not appear to be a dominant process because of the small X-ray emitting mass and the fact that the radiative shells appear to be at the outer edges of the remnants. Evolutionary calculations that include heat conduction are needed for a detailed comparison with these remnants.

Massive stars are expected to frequently have sufficient photoionizing radiation and winds to sweep out a region $\gtrsim 15 \mathrm{pc}$ in size around the stars. It is only the massive stars at the low mass end $\left(\lesssim 12 M_{\odot}\right)$ that may interact directly with a molecular cloud. Although these are a minority of the remnants, they are bright because of the interaction with dense gas and so stand out in the observational sample. The further investigation of these objects can be expected to shed light on a number of physical processes, from molecular shock emission to the high-energy $\gamma$-ray emission from cosmic rays.

I am grateful to David Bertsch and Claes Fransson for correspondence and discussion. The supernova remnant web page of David Green (http://www.mrao.cam.ac.uk/surveys/snrs/) was a useful guide to the observations. Support for this work was provided in part by NASA grant NAG-5-3057. 


\section{REFERENCES}

Asaoka, I., \& Aschenbach, B. 1994, A\&A, 284, 573

Bertoldi, F., \& McKee, C. F. 1990, ApJ, 354, 529

Bertschinger, E. 1986, ApJ, 304, 154

Blandford, R. D., \& Cowie, L. L. 1982, ApJ, 260, 625

Blandford, R. D., \& Ostriker, J. P. 1978, ApJ, 221, L29

Blitz, L. 1993, in Protostars and Planets III, ed. E. H. Levy and J. I. Lunine (Tucson: Univ. of Arizona), 125

Braun, R., \& Strom, R. G. 1986, A\&A, 164, 193

Burton, M. G., Geballe, T. R., Brand, P. W. J. L., \& Webster, A. S. 1988, MNRAS, 231, 617

Burton, M. G., Hollenbach, D. J., Haas, M. R., \& Erickson, E. F. 1990, ApJ, 355, 197

Chevalier, R. A. 1974, ApJ, 188, 501

Cioffi, D. F., McKee, C. F., \& Bertschinger, E. 1988, ApJ, 334, 252

Claussen, M. J., Frail, D. A., Goss, W. M., \& Gaume, R. A. 1997, ApJ, 489, 143

Cornett, R. H., Chin, G., \& Knapp, G. R. 1977, A\&A, 54, 889

Cowie, L. L., McKee, C. F., \& Ostriker, J. P. 1981, ApJ, 247, 908

Cox, D. P. 1972, ApJ, 178, 159

Cui, W., \& Cox, D. P. 1992, ApJ, 401, 206

de Jager, O. C., \& Mastichiadis, A. 1997, ApJ, 482, 874

de Jager, C., Nieuwenhuijzen, H., \& van der Hucht, K. A. 1988, A\&AS, 72, 259

DeNoyer, L. 1979a, ApJ, 228, L41

DeNoyer, L. 1979b, ApJ, 232, L165

DeNoyer, L. 1983, ApJ, 264, 141

Dickman, R. L., Snell, R. L., Ziurys, L. M., \& Huang, Y.-L. 1992, ApJ, 400, 203

Digel, S. W., Hunter, S. D., \& Mukherjee, R. 1995, ApJ, 441, 270

Digel, S. W., Grenier, I. A., Heithausen, A., Hunter, S. D., \& Thadeus, P. 1996, ApJ, 463, 609 
Draine, B. T., \& McKee, C. F. 1993, ARA\&A, 31, 373

Duin, R. M., \& van der Laan, H. 1975, A\&A, 40, 111

Elitzur, M. 1976, ApJ, 203, 124

Erickson, W. C., \& Mahoney, M. J. 1985, ApJ, 290, 596

Esposito, J. A., Hunter, S. D., Kanbach, G., \& Sreekumar, P. 1996, ApJ, 461, 820

Falgarone, E., \& Phillips, T. G. 1991, in Fragmentation of Molecular Clouds and Star Formation, ed. E. Falgarone, F. Boulanger, \& G. Duvert (Dordrecht: Kluwer), 119

Fesen, R. A., \& Kirshner, R. P. 1980, ApJ, 242, 1023

Frail, D. A., Goss, W. M., Reynoso, E. M., Giacani, E. B., Green, A. J., \& Otrupcek, R. 1996, AJ, 111,1651

Gaisser, T. K., Protheroe, R. J., \& Stanev, T. 1998, ApJ, 492, 219

Giacani, E. B., et al. 1997, AJ, 113, 1379

Giovanelli, R., \& Haynes, M. P. 1979, ApJ, 230, 404

Goss, W. M., \& Robinson, B. J. 1968, Ap. Lett., 2, 81

Goss, W. M., Skellern, D. J., Watkinson, A., \& Shaver, P. A. 1979, A\&A, 78, 75

Green, D. A. 1986, MNRAS, 221, 473

Harrus, I. M., Hughes, J. P., Singh, K. P., Koyama, K., \& Asaoka, I. 1997, ApJ, 488, 781

Heiles, C., Goodman, A. A. McKee, C. F., \& Zweibel, E. G. 1993, in Protostars and Planets III, ed. E. H. Levy and J. I. Lunine (Tucson: Univ. of Arizona), 125

Hollenbach, D., \& McKee, C. F. 1989, ApJ, 342, 306

Huang, Y.-L., Dickman, R. L., \& Snell, R. L. 1986, ApJ, 302, L63

Keohane, J. W., Petre, R., Gotthelf, E. V., Ozaki, M., \& Koyama, K. 1997, ApJ, 484, 350

Koo, B.-C., \& Heiles, C. 1995, ApJ, 442, 679

Kovalenko, A. V., Pynzar, A. V., \& Udal'tsov, V. A. 1994, Astron. Rep., 38, 95

Kramer, C., Stutzki, J., Röhrig, R., \& Corneliussen, U. 1998, A\&A, 329, 249

Kundu, M. R., \& Velusamy, T. 1972, A\&A, 20, 237

Long, K. S., Blair, W. P., White, R. L., \& Matsui, Y. 1991, ApJ, 373, 567 
Longair, M. S. 1994, High Energy Astrophysics, 2nd Ed., Vol. 2 (Cambridge: Cambridge Univ. Press)

McKee, C. F., \& Cowie, L. L. 1975, ApJ, 195, 715

McKee, C. F., Van Buren, D., \& Lazareff, B. 1984, ApJ, 278, L115

Moorhouse, A., Brand, P. W. J. L., Geballe, T. R., \& Burton, M. G. 1991, MNRAS, 253, 662

Mufson, S. L., McCollough, M. L., Dickel, J. R., Petre, R., White, R., \& Chevalier, R. 1986, AJ, 92,1349

Myers, P. C., \& Goodman, A. A. 1991, ApJ, 373, 509

Osterbrock, D. E. 1989, Astrophysics of Gaseous Nebulae and Active Galactic Nuclei (Mill Valley: University Science Books)

Panagia, N. 1973, AJ, 78, 929

Petre, R., Szymkowiak, A. E., Seward, F. D., \& Willingale, R. 1988, ApJ, 335, 215

Raymond, J. C. 1979, ApJS, 39, 1

Reach, W. T., \& Rho, J. 1996, A\&A, 315, L277

Reach, W. T., \& Rho, J. 1998, preprint (astro-ph/9804142)

Reynolds, S. P., \& Moffett, D. A. 1993, AJ, 105, 2226

Rho, J.-H., \& Petre, R. 1996, ApJ, 467, 698

Rho, J.-H., Petre, R., Schlegel, E. M., \& Hester, J. J. 1994, ApJ, 430, 757

Richter, M. J., Graham, J. R., Wright, G. S., Kelly, D. M., \& Lacy, J. H. 1995a, ApJ, 449, L83

Richter, M. J., Graham, J. R., \& Wright, G. S. 1995b, ApJ, 454, 277

Schaller, G., Schaerer, D., Meynet, G., \& Maeder, A. 1992, A\&AS, 96, 269

Seta, M., et al. 1998, ApJ, in press

Shull, J. M. 1980, ApJ, 237, 769

Shull, J. M., \& McKee, C. F. 1979, ApJ, 227, 131

Slavin, J. D., \& Cox, D. P. 1992, ApJ, 392, 131

Sturner, S. J., Skibo, J. G., Dermer, C. D., \& Mattox, J. R. 1997, ApJ, 490, 619

Tauber, J. A., Snell, R. L., Dickman, R. L., \& Ziurys, L. M. 1994, ApJ, 421, 570 
Turner, B. E., Chan, K.-W., Green, S., \& Lubowich, D. A. 1992, ApJ, 399, 114 van Dishoeck, E. F., Jansen, D. J. \& Phillips, T. G. 1993, A\&A, 279, 541

Wang, Z. R., Asaoka, I., Hayakawa, S., \& Koyama, K. 1992, PASJ, 44,303

Wheeler, J. C., Mazurek, T. J., \& Sivaramakrishnan, A. 1980, ApJ, 237, 781

White, R. L., \& Long, K. S. 1991, ApJ, 373, 543

Williams, J. P., Blitz, L., \& Stark, A. A. 1995, ApJ, 451, 252

Williams, J. P., \& McKee, C. F. 1997, ApJ, 476, 144

Wolszczan, A., Cordes, J. M., \& Dewey, R. J. 1991, ApJ, 372, L99

Wootten, A. 1977, ApJ, 216, 440

Wootten, A. 1981, ApJ, 245, 105 
Fig. 1. - The structure of the radiative shell for the case $B_{t} \propto \rho r$. The shock front is at $\zeta=0$. The density and pressure are proportional to $E_{o}$ and $P_{o}$, respectively, and $W_{o}$ is a measure of the deviation of the velocity from a constant. The expansion exponent of the radiative shell is $\eta=0.3$.

Fig. 2.- The same as fig. 1, except for the case $B_{t} \propto \rho$.

Fig. 3.- The evolution of the dimensionless position $\zeta_{s}$, velocity $U_{o}$, and column density $\Omega$ as a function of dimensionless time $y$ for a slab of gas driven into a molecular clump by a radiative shell. The dashed line is an analytical approximation to $\Omega$ that becomes increasingly accurate at late times. By the point $\zeta_{s}=0.453$, the reflected shock front has moved through the radiative shell and the slab continues to move by its momentum. 
Table 1. Radii of HII Regions and Wind Bubbles

\begin{tabular}{ccrccc}
\hline $\begin{array}{c}\text { Mass } \\
\left(M_{\odot}\right)\end{array}$ & Type & $\begin{array}{r}R_{\text {ionized }} \\
(\mathrm{pc})\end{array}$ & $\begin{array}{c}\dot{M} \\
M_{\odot} \mathrm{yr}^{-1}\end{array}$ & $\begin{array}{c}\tau_{m s} \\
(\mathrm{yr})\end{array}$ & $\begin{array}{c}R_{b} \\
(\mathrm{pc})\end{array}$ \\
\hline 20 & O9V & 13.6 & $1 \times 10^{-7}$ & $7 \times 10^{6}$ & 11 \\
16 & B0V & 8.0 & $6 \times 10^{-8}$ & $9 \times 10^{6}$ & 10 \\
12 & B1V & 1.6 & $6 \times 10^{-9}$ & $13 \times 10^{6}$ & 5.3 \\
10 & B2V & 1.0 & $5 \times 10^{-10}$ & $18 \times 10^{6}$ & 2.6 \\
8 & B3V & 0.5 & $1 \times 10^{-11}$ & $26 \times 10^{6}$ & 0.8 \\
\hline
\end{tabular}




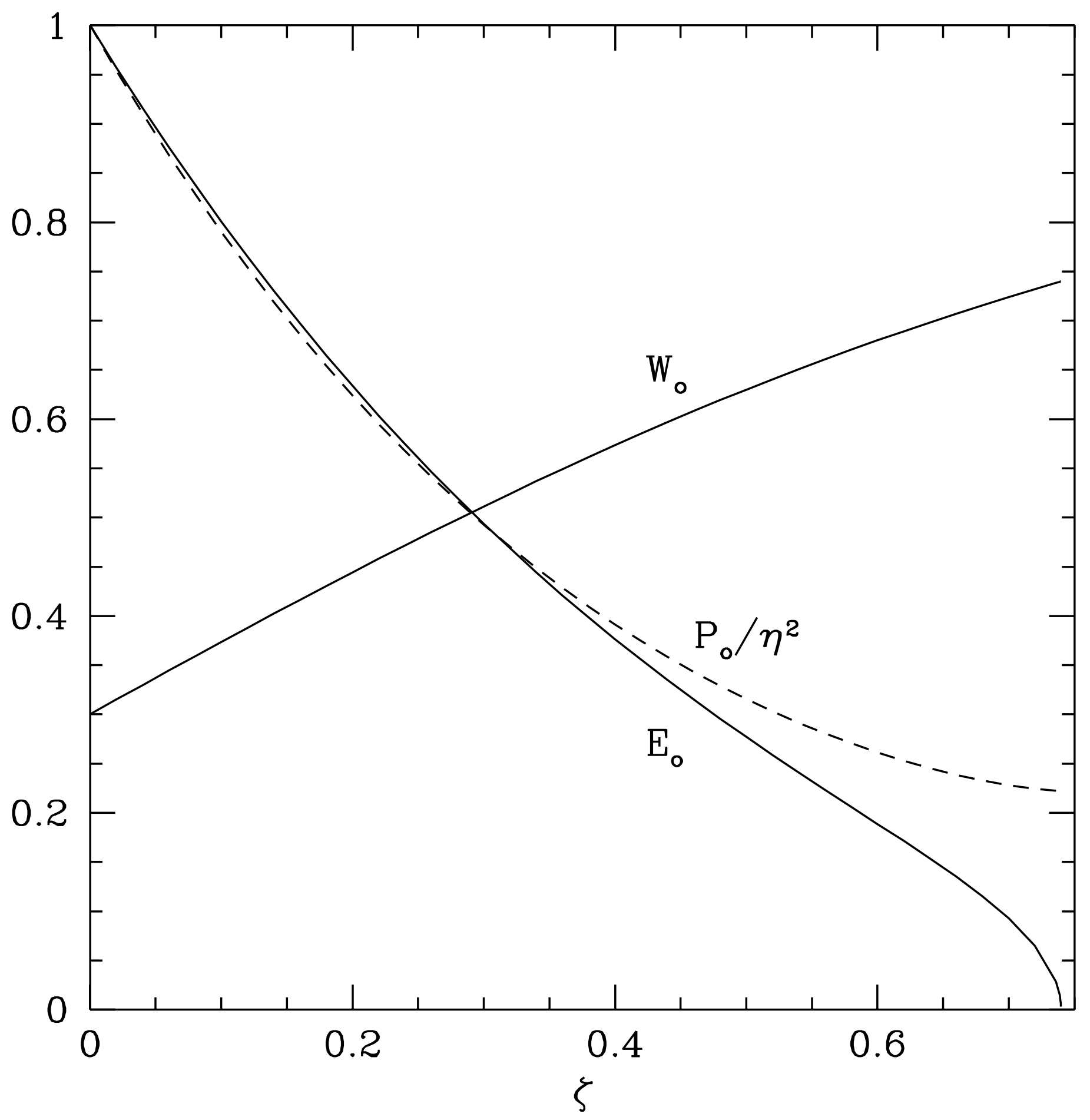




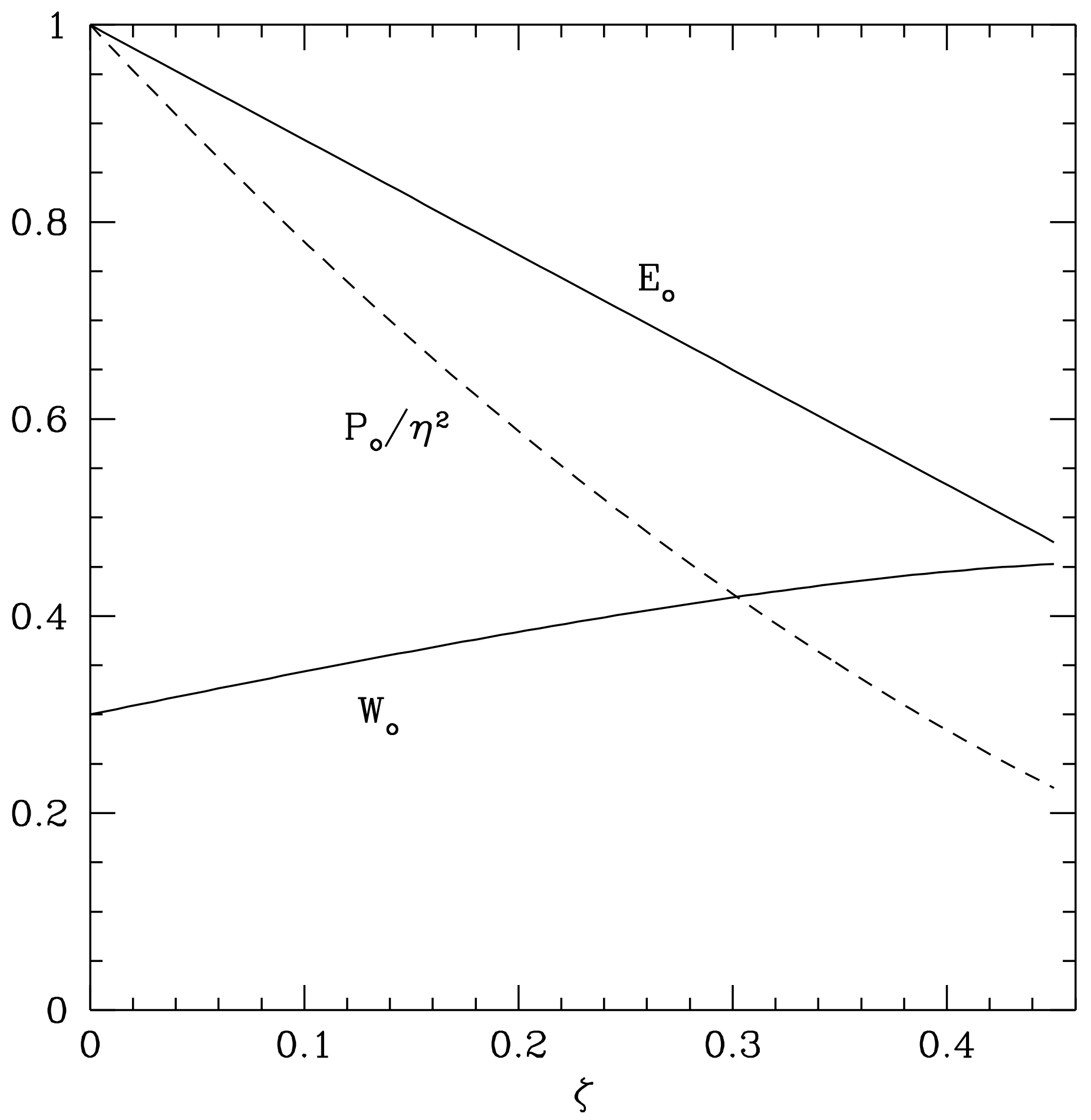




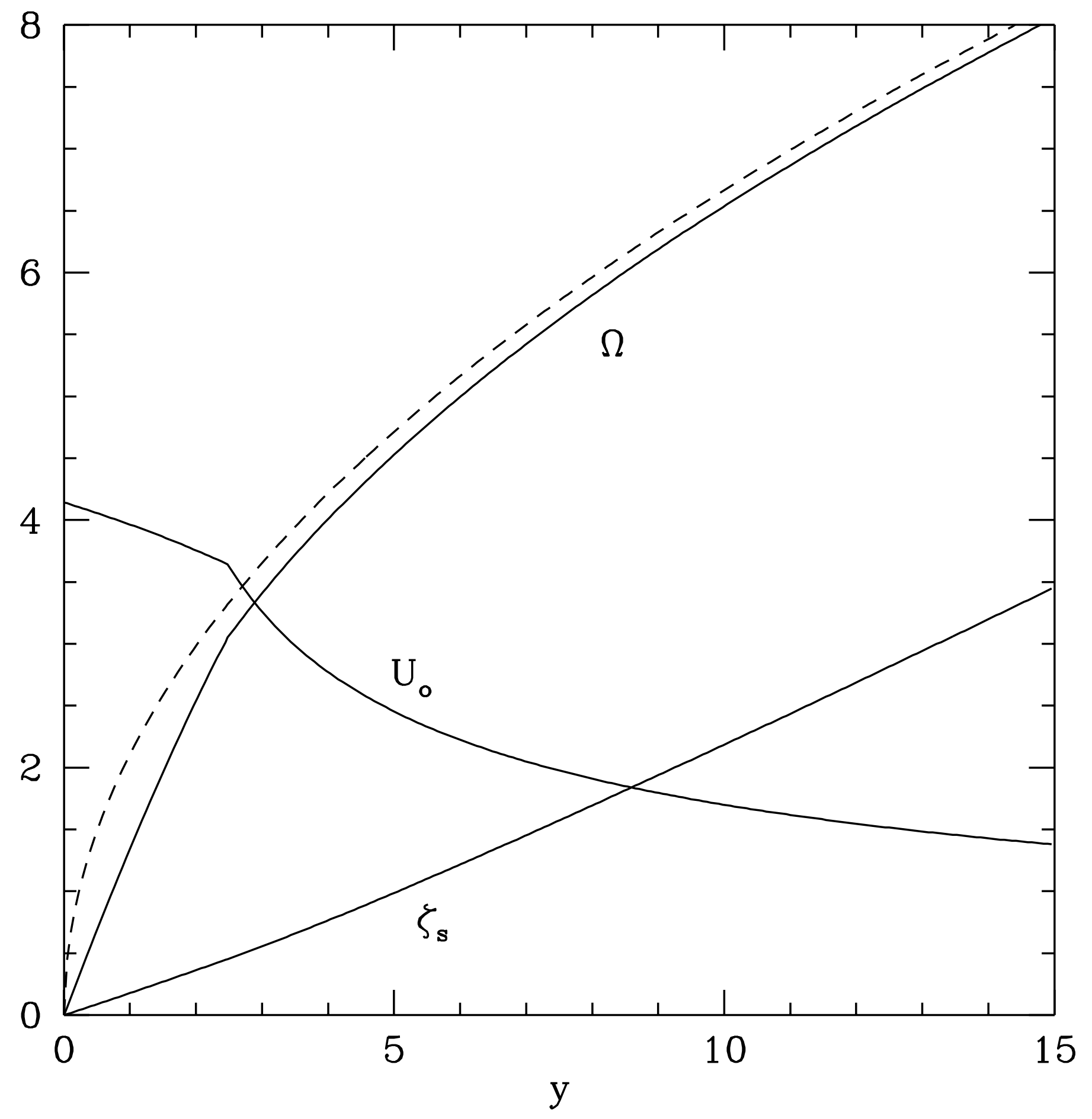

